\title{
The Use of Fractions in Public-Key Cryptosystems
}

\author{
Hartmut Isseihorst \\ Gesellschaft für elektronische informationsverarbeitung \\ Oxfordstr. 12-10, D-5300 Eonn 1 \\ West Germany
}

Abstract. This paper discusses an asymmetric cryptosystem based on fractions. the $\mathrm{R}^{\mathrm{k}}$-system, which can be implemented last using only additions and multiplications. Also it is very simple to initialize the system and to generate new keys. The $\mathrm{R}^{\mathrm{k}}$-system makes use of the difficulty to compute the numerator and the denumerator of a fraction only knowing the rounded floating point representation. It is also based on the difficulty of a simultaneous diophantine approximation with many parameters and only a little error bound.

\section{INTRODUCTION}

Many known public-key cryplosystems deal with integer problems like factorization, discre:e logarithms or knapsacks. Searching for another foundation of security we allow the use of real numbers, especially fractions.

Everyone knows that it is easy to choose wo primes $p$ and $q$ and to compute the product $n=p$. G. But up to now it is difficult to calculate the factors $p$ and $q$ only knowing $\mathrm{n}$. if $\mathrm{n}$. is greater than $10^{200}$. But knowing $\mathrm{n}$ one has enough information to compute $p$ and $q$, because factorization is deterministic. To avoid this one can try the following: Allowing real numbers it is possible to :eplace the multiplication by the division. To be more precisely, we pose the 


\section{Problem}

Let $a$ and $p$ be integers with $1<a<p<10^{1000}$ and $\operatorname{gcd}(a, p)=1$. Denote

$$
x_{n} \cdot 10^{-n} \cdot\left\lfloor 10^{n} \cdot a / p\right\rfloor, n \in \mathbb{N}
$$

1. Is it possible to compute $a$ and $p$ from $x_{n}$ with a suitable parameter $n$ ?

2. Is it possible to choose the parameter $n$ in a way such that it is impossible to calculate $a$ and $p$ from $x_{n}$ ?

The following theorem solves the problem.

\section{Theorem 1}

Let $a, p, k$ be integers with $10^{k-1}<p<10^{k}, 1<a<p, \operatorname{gcd}(a, p)=1$.

1. Only knowing $x_{2 k}$ it is easy to compute $a$ and $p$.

2. One cannot calculate $a$ and $p$ from $x_{n^{\prime}}$ if $0<\pi<2 k-50$ and $p$ is a prime.

\section{Proof:}

1. Let $0<s<t<1$ and $s=/ s_{1}, \ldots, s_{r} /, t=/ t_{1} \ldots, t_{m} /$ (the continued fractions of $s$ and $t$ ). Put formally $s_{i}=\infty$ for all $i>r$ and $t_{i}=\infty$ for all $i>m$. Then find $j$ with $s_{i}=t_{i}$ for all $i \in[1: j-1]$ and $s_{j} \neq i_{j}$. Define

$$
q= \begin{cases}s_{j+1} & j \in 2 \mathbb{N}, j \geq I \\ s_{j} & j \in 2 \mathbb{N}, j \geq I \\ t_{j}+1 & j \in 2 \mathbb{N}+1, j<m \\ t_{j} & j \in 2 \mathbb{N}+1, j \geq m .\end{cases}
$$

Then $v=/ s_{1}, \ldots, s_{j-1}, q /$ is the irreducible fraction in [s.1] with the lowest denominator ([Knuth 81, p.006]).

If $a / b$ and $c / d$ are consecutive fractions in the Farey-sequence $F_{n}, n z 2$. it holds

$$
|a / b-c / d|=\frac{1}{b d} \geq \frac{1}{n(n-1)}
$$

([Niven and Zuckeman 70, p.180])

Hence $a / p \in\left[x_{2 k}, x_{2 k}+10^{-2 k}\right] \cap F_{10 k}=\{a / p\}$.

So the algorithm acove computes $a$ and $p$ from the input $s=x_{2 k}, t=x_{2 k}-i 0^{-2 k}$.

2. If $a / p, c / d$ are consecutive fractions in $F_{p}$ we have 


$$
\left[x_{n}, x_{n}+10^{-n}\right] \cap F_{p}=\{a / p\} \Leftrightarrow 10^{-n} \leq\left|\frac{a}{p}-\frac{c}{d}\right|=\frac{1}{d p} \Leftrightarrow n \geq \log _{10}(p \cdot d)
$$

From [Horster and Isselhorsi 89, p.101] we have

$$
\frac{1}{\left|F_{p}\right|} \sum_{\substack{\text { 高}, \frac{c}{a} \in F_{p} \\ \text { consecutiv }}} \log _{10}(b \cdot d) \approx 2 \cdot \log _{10}(p+1)-\frac{1}{\log _{e}(10)} .
$$

so to compute $a$ and $p$ one needs to know $x_{n}$ with $n=2 \cdot \log _{10}(p)$ almost everytime. Knowing only $2 \cdot \log _{10}(p)-50$ digits, one has to guess 50 sequential digits following $x_{n}$ or approximately 50 partial quorients of $a / p$ ( [Isseinorst 88 , p.1041).

Here one should observe, that the probability of $a / p$ having a short period is nearly zero, because there are only a few primes q having a short perioc in $1 / q$ ([Horster and Isselhorsi 89, p. 89]).

\section{Remark}

Now it is possible to use the fraction $a / p$ with $2 \cdot l_{0} g_{10}(p)-50$ digits as a public key, because it is impossible to compute $a$ and $p$ having not enough information about $a / p$.

\section{THE PROPOSED PUBLIC-KEY CRYPTOSYSTEM}

Knowing the results about fractions we look for a way to use them for building a public-key cryptosystem. One possibility to do this is based on the computation with a real modulus:

$$
\begin{gathered}
a \equiv b(\bmod c) \Leftrightarrow(a-b) / c \in \mathbb{N}, a \cdot b, c \in \mathbb{R}^{+} \\
a \operatorname{MOD} b:=a-\lfloor a / b\rfloor \cdot b
\end{gathered}
$$

The following lemma comidines the results about fractions with a real mocuius.

\section{Lemma}

Let $p$ be a prime, : $>0, a, a^{*}:[1: p-1]$ with $a \cdot a^{*} \equiv 1(\bmod p)$ and denote

$$
\begin{gathered}
c:=: \cdot a / p \\
E(x):=(c \cdot x) M O D \text { t. } x \in[0: p-i]
\end{gathered}
$$




$$
D(y):=\left(y / t \cdot p \cdot a^{*}\right) \text { MOD } p
$$

then $D(E(x))=x$ for all $x \in[0: p-1]$.

Proof:

Since $a / r$ MOD $b / r=a / r-L(a / r) /(b / r)\rfloor \cdot b / r$ a $(a M O D b) / r$ for all $r>0$ we get

$$
\begin{aligned}
D(E(x)) & =\left((t \cdot a / p \cdot x M O D t) / t \cdot p \cdot a^{*}\right) \text { MOD } p \\
& =\left((t / p \cdot(a \cdot x M O D p)) / t \cdot p \cdot a^{*}\right) \text { MOD } p \\
& =(a \cdot x \text { MOD } p) \cdot a^{*} \text { MOD p a x. }
\end{aligned}
$$

This can be interpreted as a model of a cryptosystem, which uses the fraction $a / p$ in the encryption function $E(x)$, but uses the integer $p \cdot a^{*}$ in the decryption function $D(y)$. Here it is important to see, that the integer $p \cdot a^{*}$ is not the same as the fraction p/a.

However it works only if one uses exact arithmetic, it is possible 10 get a and $\mathrm{p}$ knowing $\mathrm{c}$. But when the system is made fault tolerant with rounded numbers, it can be secured and implemented using the results above.

So the lemma can be improved to a public-key cryptosystem, which will be discussed here:

\section{The $\mathrm{R}^{\mathrm{k}}$-System}

Assumptions: Let - $p$ be a prime, $p>10^{250}, k \in \mathbb{N}+2$

$$
\begin{aligned}
& -A=\left(a_{i, j}\right) \in \mathbb{Z}_{p}^{k \times k}, \operatorname{det}(A) \neq O(\bmod p) \\
& -A^{*}=\left(a_{i, j}^{*}\right) \in \mathbb{Z}_{p}^{k \times k} \text { with } A \cdot A^{*} \equiv I(\bmod p) \\
& -i \in(O, p)(\text { for example } t=1) \\
& \left.-z=\Gamma \log _{10}(4 \cdot p / t)\right\rceil \\
& -c_{i, j}^{n}=10^{-n}\left\lfloor 10^{n} \cdot t \cdot a_{i, j} / p\right\rfloor \cdot C_{n}=\left(c_{i, j}^{n}\right) \in \mathbb{R}^{k \times k} \text { with } \\
& \left.n=\Gamma 2 \cdot \log _{10}(p)-50-\log _{10}(t)\right\rceil
\end{aligned}
$$

Plaintext:

$$
\left.-X \in \mathbb{Z}_{\mathrm{m}}^{\mathrm{k}} \text { with } \mathrm{m} \cdot \mathrm{L} \frac{\mathrm{p}}{10^{50} \cdot 4 \mathrm{k}}\right\rfloor
$$

Encryption function: $\quad E(X)=10^{-z}\left[10^{z} \cdot\left(\left(C_{n} \cdot X\right) M O D t\right)\right\rfloor$ 
Public keys: $\quad-C_{\Omega}, t, z$

Decryption function: $\quad D(Y)=\left(A^{*} \cdot\lfloor Y \cdot p / t+1 / 2\rfloor\right)$ MOD $p$

Secret keys: $\quad-p \cdot A^{*}$

\section{Theorem 2}

The $R^{k}$-system holds $D(E(X))=X$ for all $X \in \mathbb{Z}_{m}^{k}$.

Proof (sketch):

The central step is

$$
\left.O \leq\left[\left(\sum_{j=1}^{k} c_{i, j}^{n} \cdot x_{j}\right) M O D \quad\right] \cdot \frac{P}{i}-\left[10^{-z} L 10^{z}\left(\sum_{j=1}^{k} c_{i, j}^{n} \cdot x_{j}\right) M O D t\right]\right] \cdot \frac{P}{i} \leq \frac{1}{4} .
$$

With [Isselhorst 88, p. 131-134] we also have

$$
\begin{aligned}
& \mid\left[\left(\sum_{j=1}^{k} t \cdot a_{i, j} / p \cdot x_{j}\right) \text { MOD } 1\right] \cdot p / t-\left[\left(\sum_{j=1}^{k} c_{i, j}^{n} \cdot x_{j}\right) \text { MOD } t\right] \cdot \frac{P}{t} \mid= \\
& \mid\left(\sum_{j=1}^{k} a_{i, j} \cdot x_{j}\right) \text { MOD } p-\left[\left(\sum_{j=i}^{k} c_{i, j}^{n} \cdot x_{j}\right) \text { MOD } t\right] \cdot \frac{p}{t} \mid<1 / 4 .
\end{aligned}
$$

Taking both inequalities together implies

$$
\begin{aligned}
& \left|\left(\sum_{j=1}^{k} a_{i, j} \cdot x_{j}\right) M O D p-\left[10^{-z}\left\lfloor 10^{z}\left(\sum_{j=1}^{k} c_{i, j}^{n} \cdot x_{j}\right) M O D t\right]\right] \cdot \frac{P}{t}\right|< \\
& \left|\left(\sum_{j=1}^{k} a_{i, j} \cdot x_{j}\right) M O D p-\left[\left(\sum_{j=1}^{k} c_{i, j}^{n} \cdot x_{j}\right) M O D t\right] \cdot \frac{P}{t}\right|+ \\
& \left|\left[\left(\sum_{j=1}^{k} c_{i, j}^{n} \cdot x_{j}\right) M O D t\right] \cdot \frac{P}{t}-\left[10^{-z}\left\lfloor 10^{z}\left(\sum_{j=1}^{k} c_{i, j}^{n} \cdot x_{j}\right) M O D+\right]\right] \cdot \frac{p}{t}\right|<\frac{1}{4}-\frac{1}{4}=\frac{1}{2}
\end{aligned}
$$

and finally $\left(\sum_{j=1}^{k} a_{i, j} \cdot x_{j}\right)$ MOD $p=L\left[10^{-z}\left\lfloor 10^{z}\left(\sum_{j=1}^{k} c_{i, j}^{n} \cdot x_{j}\right)\right.\right.$ MOD $\left.\left.\left.t\right]\right] \cdot \frac{p}{i}+\frac{1}{2}\right\rfloor$.

\section{DISCUSSION}

\section{a) SECURITY}

i) The $\mathrm{R}^{\mathrm{k}}$-system with farameter $k=1$ is not secure. It is easy to approximate the number $c_{n} / t \approx e / t$ by continued fractions. Then one can simulate the original 
$\mathrm{R}^{1}$-system with e/f instead of $a / p$. So one can break a $\mathrm{R}^{i}$-system without knowing the secret keys a and $p$.

ii) With $k 22$ one can try the same attack: one looks for an approximation of $c_{i, j}^{\pi / t}$ $\approx e_{i, j} / \mathrm{f}$, which is a simultaneous diophantine approximation.

But note the following lacts:

- the number of simuitaneous diophantine approximations increases quadratically with $k$

- the error bound is always very small ( $\sim 10^{50 / p^{2}}$, [Horster and Isselhorst 89])

- the common denomirator $f$ has to be bounded: $i \leq 10 \cdot p$.

Furthermore the best algorithm to solve simultaneous diophantine approximation problems of this kinc would in my opinion Lagarias' algorithm [Lagarias 85]. which uses $O\left(k^{12} \cdot\left(k^{2} \cdot \log _{2}\left(10^{n}\right)+\log _{2}(p)\right)^{4}\right)$ bit-operations to find some approximation. It is not guaranteed that solutions found by this procedure will work.

\section{b) ADVANTAGE}

i) The advantage of the $\mathrm{R}^{\mathrm{k}}$-system is, that it works fast. To encrypt and decrypt $\mathrm{k}$ integers out of $[0: \mathrm{m}]\left(\mathrm{m} \approx \mathrm{p} \cdot 10^{-50}\right)$ there are $\left(2 \mathrm{k}^{2}+10 \mathrm{k}\right)$ operations like addition, multiplication and reduction. (With $k=10, t=1$ there are 9 additions, 10 multiplications and 1 reduction to encrypt or decrypt one number). It is possible to choose $t=1$, so that the reduction mod $t$ is very simple.

ii) It is easy to initialize the $R^{k}$-system and to generate new keys, because one needs only one prime $p$ and an invertible matrix $A$ with the $\mathbb{Z}_{p}$-invers $A$.

iii) To strengthen the system one can select a higher dimension $k$ without the need to use larger rumbers as in the RSA-scheme.

iv) The $\mathrm{R}^{\mathrm{k}}$-system ercvides another way to build a public-key cipher without using the well known arithmerical problems like factorization or knapsacks.

\section{c) DISADVANTAGE}

i) The security of the $R^{k}$-system is not proved. 
ii) The size of the public and the secret key might be regarded as a disadvantage. But uniike knapsack-schemes within the $\mathrm{R}^{k}$-system one encrypts $\log _{2}(m) / k$ > $>1$ bits with every component of the key.

\section{FURTHER RESEARCH}

i) Look for other attacks for the $R^{k}$-system: One is to try to get the prime $p$ with a simultaneous diophantine approximation with only a few components of the key matrix $C_{n}$.

ii) Examine if the security of the $\mathrm{R}^{\mathrm{k}}$-system holds when $\mathrm{p}$ is an arbitrary integer and not necessarily a prime, and $\operatorname{det}(A) \neq O(\bmod p)$. So the initialization becomes easier.

iii) Examine if one can select a small number $k \in[2: 10]$, such that the $R^{k}$-system is very fast. This should be used for messages which have to be secret only for a short time (like one hour or one day e. g. in military use).

\section{CONCLUSION}

The paper shows how to use fractions in a public-key cryptosystem. which is based on the problem of a simultaneous diophantine approximation with many parameters. The rew $\mathrm{R}^{\mathrm{k}}$-system can be implemented in a fast way using only addition and muiticilication with only one reduction. Also new keys can be produced very simply, so that one can use a different pair of keys in every communication.

\section{REFERENCES}

[Horster and Issehorst 39]:

P. Horster, H. Isseihorst, Approximative Public-Key-Kryptosysteme, Informatik-Fachberichte 200 (Heideiberg: Springer, 1989) 
[Isselhorst 88]:

H. Isselhorst. Ein Beitrag zur Verwendung rationaler Zahlen in Public-Key Kryptosystemen (Heidelberg: Hüthig. 1988)

[Knuth 81]:

D. E. Knuth, The Art of Computer Programming Vol.2. Seminumerical Algorithms. 2ed (Reading: Addison Wesley, 1981)

[Lagarias 85]:

5. C. Lagarias, The Computational Complexity of Simultaneous Diophantine Approximation Problems," SIAM J. Comput. Vol.14 Nol (1985), p.196-209

[Niven and Zuckerman T6]:

l. Niven, H. S. Zuckerman, Einführung in die Zahlentheorie I (Mannheim: Bibliographisches Institut, 1970)

\section{SYMBOLS}

$\mathbb{N}=\{0,1,2,3, \ldots\} \quad \mathbb{N}+i=(i, i+1, i+2, i+3, \ldots\}$

$\mathbb{Z} \cdot(\ldots,-2,-1,0,1,2, \ldots\} \quad \mathbb{Z}_{p}=\{0,1,2, \ldots, p-2, p-1\}$

$\mathbb{R}=$ real numbers

$\lfloor x\rfloor=$ greatest integer less than $x \quad\lceil x\rceil=$ lowest integer greater than $x$ $\operatorname{gcd}(a, b)=$ greatest common diviso: of $a$ and $b$

$[a: b]=\{a, a+1, a+2, \ldots, b-1, b\}$

$I=$ unit matrix

\section{SMALL EXAMPLE}

The prime:

The dimension

The invertible matrix

The inverse matrix

$$
\begin{aligned}
& p=04301 \\
& k=2
\end{aligned}
$$

$$
\begin{aligned}
& A=\left(\begin{array}{ll}
5387 & 2993 \\
7461 & 4001
\end{array}\right) \\
& A=\left(\begin{array}{ll}
14109 & 19322 \\
59703 & 52039
\end{array}\right)
\end{aligned}
$$


the modulus

The constants

The key-matrix

Plaintext

Encryption.

Decryption: $\mathrm{t}=1$

$\mathrm{n}=9, \mathrm{z}=0$

$c_{n}=\left(\begin{array}{ll}0,083777857 & 0,0465467 \\ 0,116032410 & 0.00222298\end{array}\right)$

$\left(\begin{array}{l}x_{1} \\ x_{2}\end{array}\right) \in \mathbb{Z}_{1000}^{2} \cdot m=1000$

$E\left(\begin{array}{l}x_{1} \\ x_{2}\end{array}\right)=10^{-6} \cdot L 10^{0} \cdot\left\{\left(C_{9} \cdot\left(\begin{array}{l}x_{1} \\ x_{2}\end{array}\right)\right)\right.$ MOD 1$\left.\}\right\rfloor$

$D\left(\begin{array}{l}y_{1} \\ y_{2}\end{array}\right)=A^{-1} \cdot L\left(\begin{array}{l}y_{1} \\ y_{2}\end{array}\right) \cdot 04301+1 / 2 」 M O D 04301$

$E\left(\begin{array}{l}500 \\ 501\end{array}\right) \cdot\left(\begin{array}{l}0.20883 \\ 0.189918\end{array}\right), D\left(\begin{array}{l}0.208830 \\ 0,189918\end{array}\right)=\left(\begin{array}{l}500 \\ 501\end{array}\right)$ 